\title{
DINÂMICA DA FROTA DE VEÍCULOS QUE ESTACIONAM NO CENTRO DE LAVRAS, MG
}

\author{
Dynamics of the convoy of vehicles parking at Lavras, MG city center
}

\author{
José Otaviano de Oliveira ${ }^{1}$, Júlio Sílvio de Sousa Bueno Filho²
}

\begin{abstract}
RESUMO
Ensaios de captura-recaptura constituem técnica amostral comumente utilizada para estimar o tamanho e outros componentes da dinâmica de uma população. Neste artigo, o objetivo foi estimar a rotatividade (entrada e saída) da frota de veículos que estacionam no centro de Lavras-MG. O período estudado inclui a última semana de agosto até a segunda semana de setembro de 2002, que coincidiu com as férias semestrais. Foram utilizados modelos generalizados Poisson (log-lineares), segundo apresentado por Cormack (1989). Tais modelos requerem registros de cada indivíduo e sua história de captura anterior, compondo grupos mutuamente exclusivos com a mesma história de captura. As placas dos veículos foram utilizadas em sua marcação. Não foi detectada qualquer fonte de interação significativa entre as recapturas, o que indica uma população fechada geográfica e demograficamente. Estimativas pontuais para o tamanho da frota apresentaram valor próximo de 6.000 veículos; no entanto, os intervalos de confiança foram muito amplos. Sugere-se que modelos mais parcimoniosos (de população fechada) resultam em estimativas mais precisas do tamanho da frota.
\end{abstract}

TERMOS PARA INDEXAÇÃO: Captura-recaptura. Modelo linear generalizado. Modelo de Cormack.

\begin{abstract}
Capture-recapture techniques were used to estimate aspects of the populational dynamics of the convoy of vehicles parking at the Lavras-MG city center during the inter-term holiday period, from August 26 until September 24, 2002. We started from the open population model of Jolly-Seber. Generalized linear models were used (log-linear), according to Cormack (1989). Such models demand individual records and also its previous capture history. The vehicles' plates were used as marks. No source of significative interaction was detected, and this suggests a geographically and demographically closed population. Point estimates values were next to the 6.000 vehicles, but the confidence regions were very broad. It was suggested that parsimonious models (closed population models) should be considered to get more accurate estimates.
\end{abstract}

INDEX TERMS: Capture-recapture. Generalized linear models. Cormack's models.

(Recebido para publicação em 14 de abril de 2003 e aprovado em 18 de agosto de 2004)

\section{INTRODUÇÃO}

Tradicionalmente, a técnica de captura-recaptura tem sido aplicada em problemas de estimação do tamanho populacional e de outros parâmetros de interesse em populações biológicas. Demógrafos e epidemiologistas, entre outros, têm adotado a técnica nas pesquisas em que o tamanho populacional é de difícil contagem. A técnica consiste em marcar os indivíduos e, em seguida, tomar amostras sucessivas (recapturas) detectando os marcados. Com os dados coletados, é possível compor a história de captura de cada grupo de indivíduos em relação a sua presença ou ausência em cada amostra. No presente estudo, a história de captura dos veículos é registrada, por meio de suas placas, em um padrão de classes mutuamente exclusivas. Considerou-se a abordagem de Cormack (1989), que utiliza os modelos log- lineares para estimar componentes da dinâmica populacional. Nessa abordagem, cada elemento é individualmente marcado com marcas únicas que não podem ser perdidas. As freqüências das classes assim constituídas são consideradas variáveis aleatórias com distribuição Poisson. É possível identificar por meio desses modelos se a população sofre interação ou pode ser considerada fechada (não há adições ou deleções de novos indivíduos).

A análise dos modelos lineares generalizados é discutida em extensão por McCullagh e Nelder (1989). No contexto da análise de experimentos de capturarecaptura, o primeiro a utilizar essa metodologia foi Feinberg (1972), que analisou os dados dispostos em uma tabela de contingência multidimensional. A mode-

\footnotetext{
1. Professor EAFAJT. Mestrando em Estatística e Experimentação Agropecuária do Departamento de Ciências Exatas da Universidade Federal de Lavras/UFLA - Caixa Postal 3037 - 37200-000 - Lavras, MG. Bolsista CAPES. otaviooliver@hotmail.com
}

2. Professor Adjunto do Departamento de Ciências Exatas da UFLA. 
lagem dos parâmetros demográficos (tanto para população biológica aberta como para população fechada) foi desenvolvida por Cormack (1989) no contexto dos modelos generalizados. Pereira (1995) aplicou a mesma metodologia no estudo da dinâmica da população de jacarés na região do pantanal mato-grossense. Evans et al. (1994) apresentaram uma teoria geral para modelar os dados de captura-recaptura em população fechada.

A escolha entre tais modelos permite estimar parâmetros de rotatividade da frota de veículos que estacionam no centro de Lavras, que foi o objetivo principal quando da realização deste trabalho.

\section{MATERIAL E MÉTODOS}

A região de aplicação do ensaio denominada “Área Central” compreende a praça Dr. Augusto Silva, as três ruas paralelas até a praça Dona Josephina (Colégio Nossa Senhora de Lourdes) e suas adjacências, incluindo ainda a região do estacionamento do Hospital Vaz Monteiro. A aplicação da técnica de capturarecaptura foi efetuada da seguinte forma:

a) a primeira amostra efetuada no dia 26/08/02 a 30/08/2002 com 181 veículos (ocasião $1-\mathrm{O}_{1}$ ); b) a segunda amostra efetuada entre 02/09/02 a 06/09/02 com 118 veículos (ocasião $2-\mathrm{O}_{2}$ );

c) a terceira amostra efetuada no dia 16/09/02 a 20/09/02 com 164 veículos (ocasião $3-\mathrm{O}_{3}$ );

d) a quarta amostra efetuada 24/09/02 com 156 veículos (ocasião $4-\mathrm{O}_{4}$ ).

Com essas amostras, foram obtidas as histórias de captura apresentadas na Tabela 1 .

\section{Parâmetros do modelo de regressão e seus correspondentes biológicos}

Os coeficientes de regressão apresentam alguma combinação de fatores de interesse para estimar o tamanho da frota em estudo. Na Tabela 2 são apresentados os parâmetros correspondentes à dinâmica da frota e seus respectivos modelos a serem estudados em cada caso específico. Estes modelos são apresentados a seguir, considerando as mais diversas situações amostrais. Genericamente, os coeficientes de regressão têm correspondência direta com os parâmetros biológicos em estudo geral de populações abertas, de acordo com a Tabela 2 .

TABELA 1 - Representação de uma estrutura fatorial $2^{4}$ para a tabela de contingência representando as ocasiões $\left(\mathrm{O}_{\mathrm{i}}\right)$ e as histórias de captura.

\begin{tabular}{|c|c|c|c|c|c|}
\hline $\mathbf{O}_{1}$ & $\mathrm{O}_{2}$ & $\mathbf{O}_{3}$ & $\mathbf{O}_{4}$ & $\begin{array}{c}\text { História de } \\
\text { Captura }\end{array}$ & $\mathbf{E}\left(\mathbf{R}_{\mathrm{a}}\right)$ \\
\hline Presente & Presente & Presente & Presente & $\mathrm{m}_{0000}$ & 0 \\
\hline Ausente & Presente & Presente & Presente & $\mathrm{m}_{1000}$ & 1 \\
\hline Presente & Ausente & Presente & Presente & $\mathrm{m}_{0100}$ & 0 \\
\hline Ausente & Ausente & Presente & Presente & $\mathrm{m}_{1100}$ & 4 \\
\hline Presente & Presente & Ausente & Presente & $\mathrm{m}_{0010}$ & 0 \\
\hline Ausente & Presente & Ausente & Presente & $\mathrm{m}_{1010}$ & 6 \\
\hline Presente & Ausente & Ausente & Presente & $\mathrm{m}_{0110}$ & 3 \\
\hline Ausente & Ausente & Ausente & Presente & $\mathrm{m}_{1110}$ & 142 \\
\hline Presente & Presente & Presente & Ausente & $\mathrm{m}_{0001}$ & 0 \\
\hline Ausente & Presente & Presente & Ausente & $\mathrm{m}_{1001}$ & 1 \\
\hline Presente & Ausente & Presente & Ausente & $\mathrm{m}_{0101}$ & 5 \\
\hline Ausente & Ausente & Presente & Ausente & $\mathrm{m}_{1101}$ & 153 \\
\hline Presente & Presente & Ausente & Ausente & $\mathrm{m}_{0011}$ & 3 \\
\hline Ausente & Presente & Ausente & Ausente & $\mathrm{m}_{1011}$ & 107 \\
\hline Presente & Ausente & Ausente & Ausente & $\mathrm{m}_{0111}$ & 170 \\
\hline Ausente & Ausente & Ausente & Ausente & $\mathrm{m}_{1111}$ & . \\
\hline
\end{tabular}

Ciênc. agrotec., Lavras, v. 28, n. 5, p. 1135-1143, set.out., 2004 
TABELA 2 - Correspondência entre parâmetros do modelo de regressão e parâmetros de componentes de rotação para um experimento com $\mathrm{s}$ amostras considerando a população aberta.

\section{Coeficientes Parâmetros do Modelo Parâmetros do Modelo de Componente de Rotação}

\begin{tabular}{lcc}
\hline Media Geral & $\mathrm{u}$ & $\operatorname{Ln}\left[N p_{1} \phi_{1} p_{2} \phi_{2} \ldots p_{s-1}\left(1-\chi_{s-1}\right)\right.$ \\
Efeitos Principais & $\mathrm{u}_{1}$, & $\ln \left[\left(1-p_{1}\right) \psi_{1} / p_{1}\right]$, \\
& $\mathrm{u}_{\mathrm{i}}$, & $\ln \left[\left(1-p_{i}\right) / p_{i}\right]$, \\
Interações & $\mathrm{u}_{\mathrm{s}}$ & $\ln \left|\chi_{2} /\left(1-\chi_{2} \phi_{2}\right)\right|$ \\
& $\mathrm{u}_{12}, \mathrm{u}_{123}, \ldots \mathrm{u}_{1234} \ldots$ & $\ln \left(\psi_{2}\right), \ln \left(\psi_{3}\right), \ln \left(\psi_{4}\right), \ldots$ \\
& $\mathrm{u}_{(\mathrm{s}-1) \mathrm{s}}, \mathrm{u}_{(\mathrm{s}-2)(\mathrm{s}-1) \mathrm{s}} \ldots$ & $\ln \left(\lambda_{s-2}\right), \ln \left(\lambda_{s-3}\right), \ln \left(\lambda_{s-4}\right)$ \\
& Demais interações & Assumido como zero \\
\hline
\end{tabular}

Fonte: Cormack (1989)

As estimativas para o tamanho populacional, probabilidades de captura, sobrevivência para os períodos amostrais podem ser obtidas a partir do modelo completo e são descritas em detalhes por Cormack (1989), como se segue:

$\mathrm{p}_{\mathrm{i}}$ : probabilidade de captura de um indivíduo não marcado na i-ésima amostra,

$\phi_{1}$ : probabilidade de um indivíduo sobreviver da iésima para ( $\mathrm{i}+1$ )-ésima amostra,

$\chi_{i}$ : probabilidade de que um indivíduo não seja visto após a i-ésima amostra,

$1 / \psi_{\mathrm{i}}:$ probabilidade de que um animal vivo e não marcado na população no momento da $(i+1)$-ésima amostra estava na população no momento da i-ésima amostra, este parâmetro refere-se à entrada de novos indivíduos, de forma permanente, dentro da área pesquisada,

$1 / \lambda_{\mathrm{i}}$ : probabilidade de que um indivíduo vivo na população no momento da i-ésima amostra mas não observado em ocasiões subseqüentes, ainda esteja vivo na população no momento da (i +1$)$-ésima amostra.

As demais estimativas podem ser obtidas diretamente dos valores estimados para as frequiências de captura:

Probabilidades de Sobrevivência $\phi_{i}$;

Os parâmetros $\phi_{i}$ representam a fração de indivíduos que sobreviveram de uma ocasião de amostragem para a seguinte. Esses parâmetros não podem ser estimados na presença de migrações, mas apenas algumas combinações dessas estimativas (a menos que se use alguma restrição). Há, no entanto, delineamentos (e os respectivos modelos) específicos para estimar parâmetros de sobrevivência.

Número de Nascimentos: $\boldsymbol{B}_{i}=\boldsymbol{N}_{i}\left(\boldsymbol{1}-\boldsymbol{p}_{i}\right) \phi_{i} \psi_{i}$;

Por nascimento, entende-se a quantidade de entrada de novos indivíduos em um período amostral específico. Esse valor é diretamente proporcional ao parâmetro de entrada obtido diretamente do modelo de regressão. Nota-se que essa quantidade somente é estimável admitindo algumas restrições. É importante observar que esse modelo resulta em estimativas imprecisas de entradas e saídas temporárias.

Parâmetros de Entrada: $\lambda_{i}=\chi_{i} /\left[\phi_{i}\left(1-p_{i+1}\right) \chi_{i+1}\right]$;

As estimativas de $\lambda_{\mathrm{i}}$ representam as entradas de indivíduos de um período para o outro. Esses parâmetros somente são estimáveis com algumas restrições, já que existem mais parâmetros do que estatísticas observáveis.

As estimativas para o tamanho populacional, probabilidade de captura, sobrevivência e número de veículos novos para os períodos amostrais podem ser obtidas a partir do modelo completo. As demais estimativas podem ser obtidas diretamente dos valores estimados para as frequiências de captura. Na seção seguinte, é apresentada a conversão entre os parâmetros biológicos e os parâmetros do modelo de regressão.

\section{Matriz do delineamento amostral}

Seja $\boldsymbol{X}$ a matriz de variáveis indicadoras que representa a presença (0) e (1) ausência do indivíduo na amostra i: 


$\begin{array}{clllll} & 1 & 0 & 0 & 0 & 0 \\ 1 & 1 & 0 & 0 & 0 \\ 1 & 0 & 1 & 0 & 0 \\ 1 & 1 & 1 & 0 & 0 \\ 1 & 0 & 0 & 1 & 0 \\ & 1 & 1 & 0 & 1 & 0 \\ 1 & 0 & 1 & 1 & 0 \\ & 1 & 1 & 1 & 1 & 0 \\ 1 & 0 & 0 & 0 & 1 \\ 1 & 1 & 0 & 0 & 1 \\ 1 & 0 & 1 & 0 & 1 \\ 1 & 1 & 1 & 0 & 1 \\ 1 & 0 & 0 & 1 & 1 \\ 1 & 1 & 0 & 1 & 1 \\ 1 & 0 & 1 & 1 & 1 \\ 1 & 1 & 1 & 1 & 1\end{array}$

Por exemplo, no presente estudo, têm-se quatro ocasiões de amostragem. O primeiro vetor coluna de $X$ representa a constante do modelo de regressão proposto. Os demais vetores colunas representam as ocasiões de captura. As linhas de $\boldsymbol{X}$ representam as histórias de captura do indivíduo durante as ocasiões amostrais. Os demais parâmetros de interesse biológicos são obtidos a partir de operações nas colunas da matriz. Tem-se a = $\left(2^{s}-1\right)$ observações, qualquer modelo retirado daí pode conter no máximo $\left(2^{\mathrm{s}}-1\right)$ parâmetros. É interessante escolher aqueles que tenham algum interesse do ponto de vista da dinâmica da população. Cormack (1989) apresentou um conjunto de parâmetros de interesse possível de serem obtidos a partir do modelo de regressão por conversão ("back transformation") ou interpretado diretamente dos parâmetros de regressão.

Para estudar os componentes de rotação dos carros, será apresentada a seguir a matriz do delineamento referente ao modelo mais completo, o qual possui todos os possíveis parâmetros de interesse. A primeira coluna da matriz refere-se à constante do modelo. As quatro seguintes representam os efeitos principais (referentes aos períodos de amostragem). A quinta e sexta colunas referem-se, respectivamente, às interações de segunda e terceira ordem, construídas como produtos diretos das columas 2 e $3\left(\beta_{5}=\psi_{2}\right)$ e das colunas 1,2 e $3\left(\beta_{6}=\psi_{3}\right)$.

O produto do último pela penúltima e a última pela penúltima e antepenúltima representam as interações de saída. A última coluna representa a interação que é relativa puramente à detecção de algum comportamento em relação ao processo amostral ou armadilha. Isso é facilmente observado na matriz do modelo:

$$
\left[\begin{array}{lllll:ll:ll:l}
1 & 0 & 0 & 0 & 0 & 0 & 0 & 0 & 0 & 0 \\
1 & 1 & 0 & 0 & 0 & 0 & 0 & 0 & 0 & 0 \\
1 & 0 & 1 & 0 & 0 & 0 & 0 & 0 & 0 & 0 \\
1 & 1 & 1 & 0 & 0 & 1 & 0 & 0 & 0 & 0 \\
1 & 0 & 0 & 1 & 0 & 0 & 0 & 0 & 0 & 0 \\
1 & 1 & 0 & 1 & 0 & 0 & 0 & 0 & 0 & 0 \\
1 & 0 & 1 & 1 & 0 & 0 & 0 & 0 & 0 & 1 \\
1 & 1 & 1 & 1 & 0 & 1 & 1 & 0 & 0 & 1 \\
1 & 0 & 0 & 0 & 1 & 0 & 0 & 0 & 0 & 0 \\
1 & 1 & 0 & 0 & 1 & 0 & 0 & 0 & 0 & 0 \\
1 & 0 & 1 & 0 & 1 & 0 & 0 & 0 & 0 & 0 \\
1 & 1 & 1 & 0 & 1 & 1 & 0 & 0 & 0 & 0 \\
1 & 0 & 0 & 1 & 1 & 0 & 0 & 1 & 0 & 0 \\
1 & 1 & 0 & 1 & 1 & 0 & 0 & 1 & 0 & 0 \\
1 & 0 & 1 & 1 & 1 & 0 & 0 & 1 & 1 & 0
\end{array}\right] \times\left[\begin{array}{c}
\hat{\beta}_{0} \\
\hat{\beta}_{1} \\
\hat{\beta}_{2} \\
\hat{\beta}_{3} \\
\hat{\beta}_{4} \\
\hat{\beta}_{5} \\
\hat{\beta}_{6} \\
\hat{\beta}_{7} \\
\hat{\beta}_{8} \\
\hat{\beta}_{9}
\end{array}\right]=\left[\begin{array}{l}
\ln \hat{m}_{0000} \\
\ln \hat{m}_{1000} \\
\ln \hat{m}_{0100} \\
\ln \hat{m}_{1100} \\
\ln \hat{m}_{0010} \\
\ln \hat{m}_{1010} \\
\ln \hat{m}_{0110} \\
\ln \hat{m}_{1110} \\
\ln \hat{m}_{0001} \\
\ln \hat{m}_{1001} \\
\ln \hat{m}_{0101} \\
\ln \hat{m}_{1101} \\
\ln \hat{m}_{0011} \\
\ln \hat{m}_{1011} \\
\ln \hat{m}_{0111}
\end{array}\right]
$$


O vetor estimativas dos parâmetros $\beta$ é obtido iterativamente por procedimento de aproximação numérica.

Alguns componentes de rotação podem ser retirados diretamente dos parâmetros do modelo de regressão. Os demais podem obtidos por combinações lineares dos parâmetros do modelo e convertidos para valores estimados dos parâmetros de interesse. Alguns deles não podem ser estimados, a não ser que alguma restrição seja efetuada.

As freqüências das classes dos grupos mutuamente exclusivos estimadas contêm tais combinações na escala logarítmica. Os estimadores $\hat{\beta}_{5}$ e $\hat{\beta}_{6}$ representam as interações de entrada e $\hat{\beta}_{7}$ e $\hat{\beta}_{8}$, as de saída. $\hat{\beta}_{9}$ deverá captar algum comportamento em relação a "armadilha". Assim, a solução para a situação mais geral possível pode ser retirada diretamente da equação de regressão ou dos valores ajustados.

\section{Ajuste do modelo linear generalizado a dados com distribuição Poisson}

O algoritmo utilizado para ajustar os modelos generalizados são descritos por Nelder e Wedderburn (1972) e trata-se de um processo de estimação de quadrados mínimos reponderados iterativamente. A estimativa de $\beta$ para a $(r+1)$-ésima iteração é

$$
\hat{\beta}_{r+1}=\left(X^{\prime} W_{r} X\right)^{-1}\left[X^{\prime} W_{r}\left(X \hat{\beta}_{r}+y_{r}^{*}\right)\right]
$$

em que $\mathbf{W}_{\mathrm{r}}$ é a matriz diagonal dos valores de $\mathrm{w}_{\mathrm{i}}$ para a r-ésimo iteração e $\boldsymbol{y}^{*}$ é o vetor de pseudo-valores.

Para um determinado valor inicial $\beta_{\mathrm{r}}$ atribuído, estima-se $\beta_{\mathrm{r}+1}$ no passo seguinte. O processo termina quando o módulo da diferença entre $\beta_{\mathrm{r}+1}$ e $\beta_{\mathrm{r}}$ convergir para zero. O procedimento de ajuste foi efetuado usando o software livre R (IHAKA, e GENTLEMAN, 1996) usando o comando "glm( )", que além do ajuste, fornece a análise de "deviance" e ferramentas para diagnósticos como os resíduos da análise.

\section{Critério para análise e seleção de modelos}

Para testar a adequação do modelo linear generalizado, aplica-se na prática a diferença das "Scaled Deviance", que para a distribuição Poisson equivale a "Deviance" dos dados, confrontando esse resultado com o valor crítico da distribuição de qui-quadrado com (n-p) graus de liberdade a um nível de significância desejado. O modelo será rejeitado se o valor da deviance for superior ao valor de qui-quadrado crítico encon- trado, caso contrário, o modelo é aceito (CORDEIRO e PAULA, 1989; DEMÉTRIO, 1993).

\section{Análise de Deviance}

Se existir uma sequiência de modelos originados de um modelo maior (modelos hierárquicos), esses podem ser testados contra o modelo com maior número de parâmetros. Dessa forma, a diferença entre as "deviances" dos dois representam um valor que tem distribuição aproximada $\chi^{2}$ (CORDEIRO e PAULA, 1989).

Sejam dois modelos $\mathrm{M}_{1}$ e $\mathrm{M}_{2}$ em que $\mathrm{M}_{1}$ possui p parâmetros e $\mathrm{M}_{2}$, q parâmetros, sendo e $\mathrm{p}>\mathrm{q}$; então: $\mathrm{L}\left(\mathrm{M}_{1}\right)-\mathrm{L}\left(\mathrm{M}_{2}\right)$ (a diferença nas verossimilhanças pelos dois modelos) segue uma distribuição aproximada de qui-quadrado com p-q, graus de liberdade.

\section{Modelos considerados}

O conjunto de modelos apresentado a seguir foi escolhido de forma a representar todas as possíveis combinações entre fatores de interesse do ponto de vista da dinâmica da frota:

a) População aberta com modelo composto de entrada e saída e dependência - M1:

$$
\begin{aligned}
& g\left(\mu_{i}\right)=\beta_{0}+\beta_{1} x_{1 i}+\beta_{2} x_{2 i}+\beta_{3} x_{3 i}+\beta_{4} x_{4 i}+ \\
& +\beta_{5} x_{1 i} x_{2 i}+\beta_{6} x_{1 i} x_{2 i} x_{3 i}+\beta_{7} x_{4 i} x_{3 i}+\beta_{9} x_{2 i} x_{3 i} .
\end{aligned}
$$

Esse é o modelo mais completo do ponto de vista da parametrização proposta por Cormack (1989). Todas as combinações dos parâmetros de interesse são contempladas.

b) População aberta (somente entrada) - M2: $g\left(\mu_{i}\right)=\beta_{0}+\beta_{1} x_{1 i}+\beta_{2} x_{2 i}+\beta_{3} x_{3 i}+\beta_{4} x_{4 i}+\beta_{5} x_{1 i} x_{2 i}+\beta_{6} x_{1 i} x_{2 i} x_{3 i}$.

Nesse modelo, discute-se o efeito de entrada de novos veículos na Área central de estacionamento. Tais efeitos são estimados pela interação em ordem crescente entre os períodos amostrais.

c) População aberta (somente saída) - M3: $g\left(\mu_{i}\right)=\beta_{0}+\beta_{1} x_{1 i}+\beta_{2} x_{2 i}+\beta_{3} x_{3 i}+\beta_{4} x_{4 i}+\beta_{5} x_{4 i} x_{3 i}+\beta_{6} x_{4 i} x_{3 i} x_{2 i}$.

Da mesma forma que os modelos anteriores, trocando apenas a ordem das interações, que são tomadas da última para a primeira, como apresentado no modelo acima, são sete parâmetros para descrever a dinâmica da rotação, para quinze observações de classes mutuamente exclusivas.

d) População aberta com modelo composto de entrada e saída - M4: 


$$
\begin{aligned}
& g\left(\mu_{i}\right)=\beta_{0}+\beta_{1} x_{1 i}+\beta_{2} x_{2 i}+\beta_{3} x_{3 i}+\beta_{4} x_{4 i}+ \\
& +\beta_{5} x_{1 i} x_{2 i}+\beta_{6} x_{1 i} x_{2 i} x_{3 i}+\beta_{7} x_{4 i} x_{3 i}+\beta_{8} x_{4 i} x_{3 i} x_{2 i} .
\end{aligned}
$$

Com esse modelo, é possível estimar os parâmetros de entrada e saída. Esses parâmetros, quando significativos, apontam para uma população aberta em termos de rotação dos seus indivíduos de forma permanente. Se nenhum deles for significativo em nenhuma ordem, pode-se concluir que a população é fechada.

Um problema comum em estimação do tipo mínimos quadrados reponderados é a presença de estimativas fora do domínio admissível para os parâmetros. Tais casos foram tratados retirando-se os parâmetros correspondentes do modelo e, em seguida, efetuado um novo ajuste (CORMACK, 1989).

e) População fechada - M6:

$$
g\left(\mu_{i}\right)=\beta_{0}+\beta_{1} x_{1 i}+\beta_{2} x_{2 i}+\beta_{3} x_{3 i}+\beta_{4} x_{4 i} .
$$

Esse modelo é composto somente pelo os efeitos principais, como no exemplo descrito na seção anterior. O coeficiente da média geral representa a ligação entre tamanho populacional e as probabilidades de captura em cada período amostral.

f) População fechada com esforço constante nas amostras centrais - M7:

$$
g\left(\mu_{i}\right)=\beta_{0}+\beta_{1} x_{1 i}+\beta_{2}\left(x_{2 i}+x_{3 i}\right)+\beta_{3} x_{4 i} .
$$

Por meio desse modelo, pode-se determinar se nas diferentes ocasiões o esforço de amostragem foi uniforme ou não. No caso abordado, considerou-se a possibilidade de que as duas amostras centrais possam ser estudadas como se fossem uma só. g) População fechada com esforço constante nas três últimas amostras - M8:

$$
g\left(\mu_{i}\right)=\beta_{0}+\beta_{1} x_{1 i}+\beta_{2}\left(x_{2 i}+x_{3 i}+x_{4 i}\right)
$$

h) População fechada com esforço constante nas três primeiras amostras - M9:

$$
g\left(\mu_{i}\right)=\beta_{0}+\beta_{1}\left(x_{1 i}+x_{2 i}+x_{3 i}\right)+\beta_{2} x_{4 i}
$$

i) População fechada com esforço totalmente constante - M10:

$$
g\left(\mu_{i}\right)=\beta_{0}+\beta_{1}\left(x_{1 i}+x_{2 i}+x_{3 i}+x_{4 i}\right)
$$

No caso do modelo M10, a equação de regressão é composta apenas pelo intercepto e o coeficiente de regressão, sendo esse modelo considerado de esforço inteiramente constante.

\section{RESULTADOS E DISCUSSÃO}

Analisando o critério de deviance utilizado nas tabelas 3 e 4, nota-se que não há diferença estatística entre os modelos de população aberta e fechada. Foi selecionado o modelo de população fechada por ser mais parcimonioso e conter todos os parâmetros dentro do espaço paramétrico.

Os modelo encaixados (submodelos do modelo geral de população fechada) não são equivalentes ao modelo de esforço variável pelo teste de qui-quadrado. Portanto, o M6 ( $\mathrm{M}_{\mathrm{t}}$ de Schnabel) foi considerado o mais representativo para o conjunto de dados. No caso em estudo, não é possível aplicar modelos com quaisquer combinações de esforço constante de amostragem.

TABELA 3 - Análise de deviance para os modelos de população fechada.

\begin{tabular}{cccccc}
\hline Modelo & GL & Deviance & dif. Dev. & dif. GL & P $\left(\chi^{2}>\right.$ Dev $)$ \\
\hline M6 & 10 & 8,8937 & - & - & - \\
M7 & 11 & 16,6166 & 7,7429 & 1 & $0,0054 * *$ \\
M8 & 12 & 17,7646 & 8,7646 & 2 & $0,0125 *$ \\
M9 & 12 & 23,5391 & 14,6454 & 2 & $0,0007 * *$ \\
M10 & 13 & 23,5452 & 14,6515 & 3 & $0,0021 * *$ \\
\hline
\end{tabular}

\footnotetext{
**Significativo ao nível de $1 \%$ de probabilidade.
}

*Significativo ao nível de $5 \%$ de probabilidade. 
Verificou-se que os modelos de população aberta não diferem estatisticamente entre si, sendo o modelo M6, de população fechada, mais parcimonioso entre todos os apresentados, sendo aceito para representar o tamanho da frota.

As estimativas dos parâmetros obtidos mostramse bem ajustadas, com baixos erros padrões e altamente significativas pelo teste de qui-quadrado. Interações de segunda ordem não foram significativas, mostrando que não há nenhum comportamento em relação ao método amostral ou entrada e saída significativos.

Os valores preditos e os valores observados, bem como intervalos de confiança para as predições, são apresentados na Tabela 6 .

TABELA 4 - Análise de deviance para os modelos de população aberta comparados com o modelo M6, de população fechada.

\begin{tabular}{cccccc}
\hline Modelo & GL & Deviance & dif. Deviance & dif. GL & P $\left(\chi^{2}>\right.$ Dev $)$ \\
\hline M1 & 5 & 5,1477 & - & - & - \\
M2 & 8 & 8,2075 & 3,0638 & 3 & $0,3818^{\mathrm{ns}}$ \\
M3 & 8 & 7,9636 & 2,8159 & 3 & $0,4209^{\mathrm{ns}}$ \\
M3 & 6 & 7,4975 & 2,3496 & 1 & $0,1253^{\mathrm{ns}}$ \\
M5 & 9 & 7,9947 & 2,847 & 4 & $0,5837^{\mathrm{ns}}$ \\
M6 & 10 & 8,8937 & 3,7465 & 5 & $0,5865^{\mathrm{ns}}$ \\
\hline
\end{tabular}

ns: não significativo

TABELA 5 - Estimativas de Máxima verossimilhança dos parâmetros para o modelo de população fechada.

\begin{tabular}{cccc}
\hline Parâmetros & Estimativa & Erro padrão & $\mathbf{P}\left(\left|\mathbf{Z}_{\mathbf{c}}\right|>|\mathbf{Z}|>\right)$ \\
\hline (Intercepto) & -5.8985 & 0.6071 & $0,0000^{* *}$ \\
P1 & 3.4425 & 0.2151 & $0,0000^{* *}$ \\
P2 & 3.8814 & 0.2198 & $0,0000^{* *}$ \\
P3 & 3.5441 & 0.2158 & $0,0000^{* *}$ \\
P4 & 3.5955 & 0.2163 & $0,0000^{* *}$ \\
\hline
\end{tabular}

**Significativo ao nível de $1 \%$ de probabilidade. 
TABELA 6 - História de captura com os valores observados, preditos e intervalo de $95 \%$ de confiança para os valores preditos.

\begin{tabular}{ccccc}
\hline $\mathbf{F}_{\mathbf{a}}$ & Valor predito & Erro padrão & Limite inferior & Limite superior \\
\hline 0 & 0,00274 & 0,6073 & 0,00083 & 0,00902 \\
1 & 0,0858 & 0,4101 & 0,0384 & 0,1916 \\
0 & 0,133 & 0,4086 & 0,0597 & 0,2963 \\
4 & 4,1593 & 0,2157 & 2,7253 & 6,348 \\
0 & 0,095 & 0,4099 & 0,0425 & 0,2121 \\
6 & 2,9686 & 0,2198 & 1,9296 & 4,5673 \\
3 & 4,6043 & 0,2149 & 3,0216 & 7,0159 \\
142 & 143,951 & 0,0817 & 122,6479 & 168,9548 \\
0 & 0,1 & 0,4098 & 0,0448 & 0,2232 \\
1 & 3,1253 & 0,2193 & 2,0333 & 4,8038 \\
5 & 4,8472 & 0,2144 & 3,1841 & 7,379 \\
153 & 151,547 & 0,0797 & 129,6248 & 177,1759 \\
3 & 3,4596 & 0,2185 & 2,2543 & 5,3093 \\
107 & 108,163 & 0,0937 & 90,0096 & 129,9777 \\
170 & 167,758 & 0,0759 & 144,5645 & 194,6731 \\
. & 5245 & 0,2169 & 3429 & 8023 \\
\hline
\end{tabular}

\section{CONCLUSÕES}

a) $\mathrm{O}$ modelo que melhor se ajustou aos dados foi o de independência entre as ocasiões amostrais, mostrando que a população é fechada, não havendo entradas ou saídas significativas no período do estudo;

b) A melhor estimativa do número de veículos que estacionam no centro de Lavras-MG foi de 5840, com intervalo de confiança de $95 \%$ de [4024; 8618] veículos.

\section{REFERÊNCIAS BIBLIOGRÁFICAS}

CORDEIRO, G. M.; PAULA, G. A. Modelos de regressão para análise de dados univariados. In: COLÓQUIO BRASILEIRO DE MATEMÁTICA, 17., 1989, Rio de Janeiro. Anais... Rio de Janeiro: IMPA-CNPq, 1989.
CORMACK, R. M. Log-linear models for capturerecapture. Biometrics, Alexandria, n. 45, p. 395-413, 1989.

DEMÉTRIO, C. G. B. Modelos lineares generalizados na experimentação agronômica. In: SIMPÓSIO DE ESTATÍSTICA APLICADA À EXPERIMENTAÇÃO AGRONÔMICA, 5.; REUNIÃO ANUAL DA REGIÃO BRASILEIRA DA SOCIEDADE INTERNACIONAL DE BIOMETRIA, 38., 1993, Porto Alegre. Anais... Porto Alegre: [s.n.], 1993.

EVANS, M. A.; BONETT, D. G.; McDONALD, L. L. A general theory for modeling capture-recapture data from a closed population. Biometrics, Alexandria, n. 50, p. 396-405, 1994. 
FEINBERG, S. E. The multiple recapture census for closed populations and incomplete $2^{\mathrm{k}}$ contingency tables. Biometrics, Alexandria, n. 59, p. 591-603, 1972.

IHAKA, R.; GENTLEMAN, R. R. A language for data analysis and grafics. Journal of computation and graphics statistics, Alexandria, v. 5, n. 3, p. 299-314, 1996.

McCUlLAGH, P.; NELDER, J. A. Generalized linear models. 2. ed. London: Chapman and Hall, 1989. 511 p.
NELDER, J. A.; WEDDERBURN, R. W. M. Generalized linear models. Journal of the Royal Statistical Society A, London, v. 135, n. 3, p. 370384, 1972.

PEREIRA, J. E. Análise de experimento de captura-recaptura através de modelos log-lineares. 1995. 106 f. Dissertação (Mestrado) - Escola Superior de Agricultura de Luiz de Queiroz, Piracicaba, 1995. 